\title{
Prevalence of intestinal parasitosis in patients afferent to the Hospital of Desio (Monza e Brianza), Italy
}

\author{
Claudia Ballabio', Natascia Venturi', Maria Roberta Sala', Paolo Mocarelli', Paolo Brambilla',2 \\ I Servizio Universitario di Medicina di Laboratorio Ospedale di Desio, Desio (MI) \\ 2 DIMS, Facoltà di medicina e Chirurgia, Università di Milano Bicocca, Milano
}

Key words: Bowel parasitosis, Coproparasitological exam, Enterobius vermicularis

Prevalenza delle parassitosi intestinali in pazienti afferenti al Presidio Ospedaliero di Desio (Monza e Brianza)

\section{SUMMARY}

From may 2008 to June 2010 we have analyzed faecal samples of 2306 subjects: in the subjects was performed the coproparassitological standard examination and in 523 of them was performed scotch test.

Of the 2306 subjects examined, I0I resulted positive, 59 to the coproparasitological exam, 43 to scotch test, and $\mathrm{I}$ to both of them (Taenia spp.). In three cases we have found positivity for two different parassitological species. Pathogenic parasites were found in 77 cases, 61 autoctones and 16 immigrates. The helminths found were Enterobius vermicularis, the prevalent, and Taenia spp., Ascaris lumbricoides, Diphillobothrium latum, Trichuris Trichiura, while the Protozoa were Giardia duodenalis and Entamoeba histolytica/dispar. Non pathogenic species were found in 27 cases, 15 among autoctones and 12 among immigrates: in detail Entamoeba coli, Endolimax nana, Blastocystis hominis. The total prevalence of parassitosis is low (4,2\%), 20,0\% in immigrate subjects and $3,4 \%$ in native subjects. Our results show that the 0-10 age group have an higher prevalence for Enterobius vermicularis. Some considerations are related for improving the complete or more exhaustive diagnosis concerning the intestinal parasitosis.

\section{INTRODUZIONE}

Le affezioni di origine parassitaria non hanno mai perso la loro attualità e la loro importanza. Anzi, in termini generali la richiesta di analisi parassitologiche ai laboratori di microbiologia clinica tende ad aumentare; questo andamento sembra essere destinato a confermarsi anche in futuro per molte ragioni. Innanzitutto le malattie di importazione di origine parassitologica sono in aumento a causa dei frequenti viaggi effettuati da un pubblico sempre più vasto nei paesi tropicali; in secondo luogo si riscontra un numero sempre maggiore di rifugiati o bambini adottati affetti da parassitosi endemiche che trovano accoglienza nel nostro paese $(1,6,7,8)$. Altre due cause dell'aumento delle parassitosi sono rappresentate dall'immunodepressione che costituisce l'attecchimento di parassiti particolari (come ad esempio Cryptosporidium o microsporidi) e per ultima causa, ma non di minore importanza, i comportamenti alimentari nuovi, soprattutto legati al consumo di alimenti crudi (ad esempio l'insorgenza di botriocefalosi o difillobotriosi dovuta al consumo di pesce crudo infetto) (11).

Inoltre le parassitosi del tratto gastrointestinale tendono a cronicizzarsi a causa della non specificità dei sintomi e della conseguente mancata diagnosi sia clinica sia microbiologica, con conseguenze potenzialmente gravi, sia pur raramente, per la salute del paziente. La maggior parte dei parassiti è ubiquitaria con prevalenza maggiore nelle zone più povere del globo a causa dell'estrema indigenza e delle problematiche ad essa correlate; tuttavia anche nei paesi industrializzati (Italia compresa) le parassitosi intestinali non sono del tutto scomparse $(1,6,9)$. Attualmente le parassitosi intestinali autoctone nel nostro paese sono piuttosto rare con una prevalenza media che, secondo i dati riportati al XXXVIII Congresso Nazionale AMCLI, si aggira attorno al 5\%, relativamente peraltro ad un singolo lavoro relativo all'area emiliana (9). Le prevalenze risultano essere basse per quanto riguarda gli elminti, tra $\mathrm{i}$ quali i nematodi Enterobius vermicularis e Strongyloides stercoralis sembrano rivestire un ruolo di una certa importanza, il primo in particolare nei bambini in età scolare ed il secondo nei soggetti anziani, ma per i quali l'approccio metodologico diagnostico deve essere mirato $(5,7,8)$. Per quanto riguarda i protozoi, Giardia duodena-

\section{Corresponding author: Claudia Ballabio}

Servizio Universitario di Medicina di Laboratorio, Ospedale di Desio

Via Mazzini I, 20033 Desio (Mi) - Tel.: 0362383296

E-mail: cballabio@uds.unimib.it 
lis e Dientamoeba fragilis rappresentano i principali protozoi patogeni intestinali presenti in Italia sebbene la loro prevalenza venga spesso sottostimata, sia per la non stretta specificità dei sintomi, sia per un errato approccio operativo diagnostico $(2,3)$. Tra i protozoi viene inoltre segnalato piuttosto frequentemente il saprofita opportunista Blastocystis hominis, il cui ruolo patogeno è tutt'ora controverso $(1,4,7)$. La segnalazione, infine, di protozoi non patogeni (amebe e flagellati) è solitamente occasionale, soprattutto in virtù delle positive abitudini alimentari acquisite nel tempo nel nostro paese e degli elevati standard igienici ormai raggiunti più o meno ovunque $(6,9,10)$.

Alla luce di quanto detto, la ricerca di parassiti fecali risulta utile nelle seguenti categorie di soggetti: soggetti portatori di disturbi intestinali aspecifici presenti da lungo tempo; pazienti con diarrea verosimilmente e presumibilmente infettiva ("diarrea del viaggiatore" in particolare), soggetti con ipereosinofilia e/o con anemia, ed infine soggetti asintomatici ma con almeno uno dei seguenti fattori di rischio: provenienza o rientro da località endemiche per parassitosi; individui a contatto stretto con soggetti dimostratisi parassitati (scuole, famiglie, collettività chiuse); anziani da sottoporre a terapie cortisoniche o immunosoppressive (in particolare per la ricerca mirata di $S$. stercoralis); pazienti immunocompromessi $(2,7)$. L'obiettivo di questo studio è valutare la prevalenza delle parassitosi nei pazienti afferenti al Presidio Ospedaliero di Desio con distinzione tra soggetti autoctoni ed immigrati, valutare l'andamento delle prevalenze nel tempo e verificare dai risultati ottenuti se vi è una prevalenza troppo inferiore rispetto ai dati in letteratura rendendo così necessaria una rivalutazione delle metodologie fino ad ora utilizzate allo scopo di migliorare la qualità diagnostica.

\section{MATERIALI E METODI}

Nel presente studio sono stati analizzati i campioni fecali pervenuti al laboratorio di Microbiologia del Presidio Ospedaliero di Desio nel periodo compreso tra Maggio 2008 e Giugno 2010.

I soggetti analizzati sono stati 2306, sia esterni residenti o domiciliati nel comune di Desio e comuni limitrofi, sia soggetti ricoverati, autoctoni e immigrati. 1783 soggetti si sono presentati al laboratorio solo con richiesta di esame coproparassitologico (ECPS), 308 solo con richiesta di Scotch test e 215 con la duplice richiesta di entrambi.

I campioni sono stati raccolti secondo le istruzioni operative del laboratorio le quali prevedono innanzitutto la raccolta delle feci a distanza di almeno 2 settimane dopo la somministrazione di antibiotici e 5-10 giorni dopo la somministrazione di bario per possibile interferenza, e quindi la raccolta di una quantità sufficiente di campione pari al volume di una noce. Vengono raccolti tre campioni di feci conservati in formalina al $10 \%$ prelevati in tre giorni differenti per effettuare l'esame coproparassitologico standard (ECPS) il quale prevede: osservazione macroscopica a fresco del campione per ricerca di eventuali proglottidi di tenie o vermi adulti, esame microscopico diretto e dopo colorazioni estemporanee (soluzione iodata, essenzialmente) utile per evidenziare le uova di elminti ma soprattutto le cisti e i trofozoiti di protozoi $(5,7)$. I campioni in formalina sono stati trattati con etilacetato, filtrati mediante l'utilizzo di Paraprep-L Formalin Kit (Diamondal) e centrifugati per 5 minuti a $1500 \mathrm{rpm}$ per l'osservazione microscopica diretta a fresco o dopo colorazione (2).

Non sono state eseguite colorazioni permanenti (Giemsa o altre) per la ricerca di D. fragilis (2, 3, $6,7)$ né colorazioni all'acido-alcool resistenza per la ricerca di Cryptosporidium spp. $(2,3)$.

I campioni di feci non conservati in formalina sono stati ritenuti non idonei per l'analisi e fatti ripetere.

La ricerca mirata di E. vermicularis, è stata effettuata in 523 soggetti sui 2306 esaminati, per lo più bambini, mediante l'utilizzo della tecnica dello Scotch Test che prevede la preparazione di almeno 3 vetrini poiché la difficoltà di esecuzione può dare false negatività (5).

\section{RISULTATI}

$\mathrm{Su}$ un totale di 2306 soggetti, 101 (4.3\%) sono risultati positivi, 59 su 1998 all'esame coproparassitologico standard (2,95\%), 43 su 523 allo scotch test $(8.20 \%)$; un soggetto è risultato positivo ad entrambi per uova di Taenia spp. (conteggiato soltanto nell'ECPS).

La prevalenza nei soggetti di sesso maschile risulta essere del 4.4\% (46 soggetti su 1026), nei soggetti di sesso femminile del 4.2\% (55 soggetti su 1280 ), del $3.4 \%$ nei soggetti autoctoni ( 75 su 2186 soggetti) e del $20 \%$ nei soggetti immigrati (26 su 120 soggetti). Nel gruppo dei soggetti parassitati, 98 risultano infetti da una sola specie parassitologica $(97 \%)$ mentre in 3 soggetti è stata riscontrata una positività per due specie parassitologiche differenti $(3 \%)$, nel particolare una specie patogena in associazione con una non patogena. Complessivamente nei 101 soggetti parassitati sono stati riscontrati 104 parassiti tra i quali 77 specie patogene $(76.6 \%)$ e 27 non patogene o di dubbia patogenicità $(26.7 \%)$.

Le specie parassitarie individuate sono così rappresentate sul totale dei positivi: $51.4 \%$ (54 casi) elminti (E. vermicularis, Taenia spp., Ascaris lum- 
bricoides, Diphillobothrium latum, Trichuris trichiura), 22.3\% protozoi patogeni (23 casi) ( $G$. duodenalis, Entamoeba histolytica/dispar) e $26.2 \%$ (27 casi) protozoi non patogeni o di dubbia patogenicità (Entamoeba coli, Endolimax nana, Blastocystis hominis).

Su un totale di 54 casi di elmintiasi, 8 sono rappresentati da soggetti autoctoni nei quali è stata riscontrata la presenza di uova di Taenia spp. in 7 casi e di D. latum in un unico caso; in 4 su 7 casi di teniasi, dall'osservazione macroscopica dei campioni è risultata anche la presenza di proglottidi (non si è peraltro proceduto all'identificazione di specie). In 2 casi di infezione da $E$. coli su un totale di 15 è stata riscontrata anche la presenza di trofozoiti.

La casistica dettagliata di tutte le positività con la relativa classificazione e distribuzione è mostrata in Tabella 1.

La distribuzione per classi di età rappresentata in Figura I mostra una percentuale maggiore di casi di parassitosi nella fascia di età $0-10$ anni (44 parassiti su 103 ) dei quali il $71.7 \%$ è rappresentato da $E$. vermicularis. Infatti la prevalenza di questo elminta risulta essere del 3.3\% (33 su 985 soggetti) in soggetti da 0 a 10 anni e dello $0.8 \%$ (11 su 1321 soggetti) in soggetti con età maggiore di
10 anni. Le positività per E. vermicularis peraltro aumentano sensibilmente se si valutano esclusivamente i risultati ottenuti con la tecnica dello scotch test, che peraltro è quella raccomandata (vedi sopra).

\section{DISCUSSIONE}

Dai risultati ottenuti in questo studio effettuato sulla popolazione afferente al Presidio Ospedaliero di Desio, emerge che la prevalenza delle parassitosi risulta essere piuttosto bassa (4.51\%): il 3.34\% dei 2306 soggetti analizzati risulta essere parassitato da elminti o protozoi di patogenicità indiscussa mentre il restante $1.17 \mathrm{da}$

Tabella I. Classificazione e distribuzione delle specie parassitologiche patogene e non o di dubbia patogenicità riscontrate nei 101 soggetti parassitati raggruppati per provenienza

\begin{tabular}{|c|c|c|c|c|c|c|}
\hline \multirow{3}{*}{ PARASSITI PATOGENI } & \multicolumn{6}{|c|}{ SOGGETTI PARASSITATI } \\
\hline & \multicolumn{2}{|c|}{ AUTOCTONI (*) } & \multicolumn{2}{|c|}{ IMMIGRATI (*) } & \multicolumn{2}{|c|}{ TOTALE (*) } \\
\hline & numero & $\%$ & numero & $\%$ & numero & $\%$ \\
\hline Enterobius vermicularis & 38 & 1.7 & 6 & 4.1 & 44 & 1.87 \\
\hline Taenia species & 7 & 0.3 & 0 & 1 & 7 & 0.3 \\
\hline Ascaris lumbricoides & 0 & 1 & $\mathrm{I}$ & 0.83 & $T$ & 0.04 \\
\hline Diphyllobothrium latum & $\mathrm{I}$ & 0.04 & 0 & 1 & $T$ & 0.04 \\
\hline Trichuris trichiura & 0 & l & I & 0.83 & 1 & 0.04 \\
\hline TOTALE ELMINTI & 46 & 2.1 & 8 & 6.6 & 54 & 2.34 \\
\hline Entamoeba histolytica/dispar** & $\mathrm{I}$ & 0.04 & $\mathrm{I}$ & 0.83 & 2 & 0.08 \\
\hline Giardia duodenalis & 14 & 0.6 & 7 & 5.8 & 2 & 1.0 \\
\hline \multicolumn{7}{|l|}{ TOTALE PROTOZOI } \\
\hline (vs PATOGENI) & 15 & 0.7 & 8 & 6.6 & 23 & 1.0 \\
\hline \multicolumn{7}{|l|}{ TOTALE PARASSITI } \\
\hline (vs) PATOGENI** & 61 & 2.8 & 16 & 13.3 & 77 & 3.34 \\
\hline Entamoeba coli & 4 & 0.13 & II & 8.4 & 15 & 0.65 \\
\hline Endolimax nana & 9 & 0.41 & $\mathrm{I}$ & 0.83 & 10 & 0.43 \\
\hline Blastocystis hominis & 2 & 0.09 & 0 & l & 2 & 0.08 \\
\hline \multicolumn{7}{|l|}{ TOTALE PARASSITI NON } \\
\hline PATOGENI (di fatto protozoi) & 15 & 0.68 & 12 & 10.0 & 27 & 1.17 \\
\hline TOTALE PARASSITI & 76 & 3.47 & 28 & 23.3 & 104 & 4.5 \\
\hline
\end{tabular}

(*) Soggetti sottoposti ad esame parassitologico: autoctoni 2186, immigrati 120, per un totale di 2306.

(**) Pur facendoli rientrare tra i sospetti verosimilmente (vs) patogeni non è stato possibile discriminare tra la forma patogena (E. histolytica) e quella non patogena (E. dispar) 
protozoi di dubbia patogenicità o sicuramente non patogeni.

I dati del presente studio trovano riscontro e confermano ulteriormente quelli presenti in letteratura; nella nostra indagine la fascia di età più interessata dalle infezioni parassitologiche intestinali risulta essere quella compresa tra 0 e 10 anni, soprattutto per quanto riguarda $E$. vermicularis, che rappresenta la specie più frequentemente associata alle parassitosi nei bambini $(1,5,7)$.

43 casi su 44 (97.7\%) di E. vermicularis sono stati identificati mediante la tecnica dello Scotch test (43 positivi su 523 scotch test effettuati, pari all' $8.2 \%$ ) e solo in un caso sono state reperite uova mediante osservazione di un preparato di feci dopo concentrazione con etilacetato (1 caso su 1998 ECPS eseguiti: 0,05\%!) Per quanto riguarda i protozoi, $G$. duodenalis rappresenta il parassita maggiormente riscontrato con 21 casi su 104 totali $(20 \%)$ che risultano uniformemente distribuiti nelle diverse classi di età.

L'assenza di positività per specie parassitologiche di indiscusso valore patogeno quali $D$. fragilis o Cryptosporidium spp., difficilmente individuabili dall'osservazione del campione a fresco senza aver effettuato colorazioni permanenti, suggerisce l'opportunità di introdurre la ricerca specifica dell'antigene per Cryptosporidium spp. mediante metodo immunocromatografico rapido (o colorazione permanente all'acido-alcool resistenza) e la colorazione di Giemsa per la ricerca di $D$. fragilis $(3,7)$. Infatti, come ribadito dalla letteratura, risulta necessario impostare nel settore di parassitologia un'attività operativa organizzata e ragionata, affinché siano applicate tutte le metodologie diagnostiche disponibili e percorsi codificati, secondo le linee guida nazionali ed internazionali $(1,2,3,7,9,10,12)$.

In fine il non reperimento di alcun caso di S. stercoralis fa riflettere sulla necessità di utilizzare tecniche specifiche per la sua individuazione (1, $2,3,7)$.

Lo studio in questione rappresenta una prima indagine per la valutazione dell'entità delle parassitosi nell'area di Desio e comuni limitrofi ed un primo step per una migliore qualificazione della diagnostica ed una più attenta sorveglianza del fenomeno.

\section{RINGRAZIAMENTI}

Si ringrazia il personale tecnico per il contributo fornito.

\section{BIBLIOGRAFIA}

1. Bernieri F, Galli D, Crotti D, Del Sante M. Studio policentrico dell'Associazione Microbiologi Clinici Italiani - Comitato di Studio di Parassitologia (AMCLI - CoSP) sulla diffusione delle parassitosi intestinali in Italia: parassitosi intestinali nell'immigrato. Giorn It Med Trop 1996; 1: 97-9.

2. Bernieri F, Crotti D, Galli D, Raglio A. Manuale illustrato di diagnostica parassitologica. Pavia: Edizioni Selecta Medica 2001.

3. Bernieri F, Casella P, Crotti D, et al. Linee Guida Operative per la Diagnosi delle Parassitosi intestinali. Microb Med, 2005; 20: 39-46.

4. Cancrini G. Parassitologia medica illustrata. Roma: Lombardo editore 1996.

5. Crotti D, D’Annibale ML. Enterobiasi nel biennio 2002-2003 nel perugino: considerazioni non soltanto diagnostiche. Le Infezioni in Medicina, 2006; 14: 92-8.

6. Crotti D, D’Annibale ML, Basileo M, La Torre G. Sondaggio preliminare copro-parassitologico in una zona andina del Perù. Le Infezioni in Medicina, 2007; 15: 181-6.

7. Crotti D. Parassitosi intestinali autoctone nella II metà degli anni '90: considerazioni critiche diagnostiche. Microb Med, 2001; 17: 7-13.

8. Guidetti C, Capatti C, Ricci L, Vecchia L. Intestinal parassitosis in Reggio Emilia during 2007: critical analysis of results. Microb Med, 2009; 24: 100-2.

9. Guidetti C, Ricci L, Vecchia L. Prevalenza delle parassitosi intestinali a Reggio Emilia e provincia nel corso del 2009. Le Infezioni in Medicina, 2010; 18: 154-61.

10. Jeong-Weon Huh, Su-Gyeong Moon, Young-Hee Lim. A survey of Intestinal Protozoan Infections among Gastroenteritis Patients during a 3-Year Period (2004-2006) in Gyeonggi-do (Province), South Korea. Korean J Parasitol, 2009; 47: 303-5.

11. Peduzzi R, Wicht B. Risorgenze parassitologiche: il caso della difillobotriosi. Biol Ital, 2010; 5/6: 15-20.

12. Wang CW, Lee YF, Chang CC, et al. Current status of Enterobius vermicularis infection in primary schoolchildren in Miaoli Country and Taichung Country, Taiwan. J Microb Immunol Infect, 2009; 42: 420-6. 